\title{
A Review on Applications of NLP with Artificial Neural Networks
}

\author{
M. Hema Sundar ${ }^{1}$, N. Thirupathi Rao ${ }^{2}$ \\ ${ }^{1,2}$ Department of Computer Science and Engineering, Vignan's Institute of \\ Information Technology (A), Visakhapatnam-530049, AP, India \\ ${ }^{2}$ nakkathiru@gmail.com
}

\begin{abstract}
Artificial neural networks are one of the more advanced and mostly used areas in research now a days. It is being used by most of the researchers in the current cutting edge technologies and their applications. Most of the researchers are trying using this part of application in these days. The usage of natural language processing and its applications are being used very high in research. In the current article, an attempt has been made to project and give a light on some of the applications where the combination of both areas was being utilized. The details about those applications and their related data were presented in detail.
\end{abstract}

Keywords: Neural networks, artificial neural networks, natural language processing, applications, text, classification, categorization.

\section{Introduction}

Neural networks are the most important mostly used techniques for identifying the different patterns or the different designs like human brain model. The data that was collected and processed from various sensors was important and done through a machinery process. The clustering of the input which was raw in form was also processed. Some of the examples of the patterns or the designs that can be identified or can be processed are the sound, images, image to text or image to sound or from sound to some images etc. All these sort of translations can be done by using the current technologies in detail[1].

The major work or the major applications and facilities provided by these networks include the classifying of data and clustering of the data and its related components. The data can be stored or processed by using this sort of various applications and its related things. Both clustering of data and classification of data can be done in the same set of applications. The features of various applications and algorithms also can be sued to verify and realter the things based on the requirement of the users or the applicants. Logical computers which were using the neural networks and its related applications will work on real numbers. These are the numbers that people use for their regular usage and for their regular calculations. The probability type of applications also can be used for these applications [2][3]. Usage of both probability theory and its related theorems and logical functions can be used for the development and usage of these methods. The logical functions also to be used to reduce the problems of repetition and more number of logical errors and logical issues.

The other important method or the logic that can be sued was the fuzzy logic. The characteristics of the users and their functions and applications can be sued mostly by the help of these logical functions and applications. The fuzzy logic can be used for many applications like the true or false type of applications and whether to do or not to applications. Washing machines are the best examples for these sorts of applications in real time world where people are directly using this sort of applications. Rule bases algorithms and rule based systems and rule based inference systems also can be processed by using this sort of applications.

Article history:

Received (March 19, 2019), Review Result (May 30, 2019), Accepted (April 6, 2019) 
Natural language processing (NLP) is the capacity of a PC program to comprehend human language as it is spoken. NLP is a part of man-made brainpower (AI) [7][8]. The advancement of NLP applications is testing since PCs customarily expect people to "talk" to them in a programming language that is exact, unambiguous and profoundly organized, or through a predetermined number of unmistakably articulated voice directions. Human discourse, be that as it may, isn't constantly exact - it is frequently vague and the semantic structure can rely upon numerous unpredictable factors, including slang, territorial vernaculars and social setting [4][5]. Punctuation and semantic investigation are two fundamental procedures utilized with common language handling. Punctuation is the game plan of words in a sentence to bode well. NLP utilizes sentence structure to survey importance from a language dependent on linguistic principles. Linguistic structure methods utilized incorporate parsing (syntactic examination for a sentence), word division (which partitions an enormous bit of content to units), sentence breaking (which spots sentence limits in huge writings), morphological division (which partitions words into gatherings) and stemming (which partitions words with expression in them to root frames) [6].

\section{APPLICATIONS OF NLP WITH ANN}

The combination of both neural networks with natural language processing models will give us more accurate and best output based performance of the systems. Some fo them are,

Data Classification and Categorization

Part of Speech Tagging

Semantic based question answering

Data detection

Language and paragraphs detection

Translation of machines data

\subsection{Data Classification and Categorization}

The classification of data and categorization of data is the major and important part in the several applications of the society. Some of them are searching of the web for some data of interest, filtering of data which was not required for the processing or for other purposes during the processing of the applications, identification of various keywords for identifying the language and its related issues, assessment of the data etc,. These applications are used in various scenarios like the identification of the negative reviews and positive reviews for a movie in the market such that more number of people can be moved towards watching that particular object or a movie. 


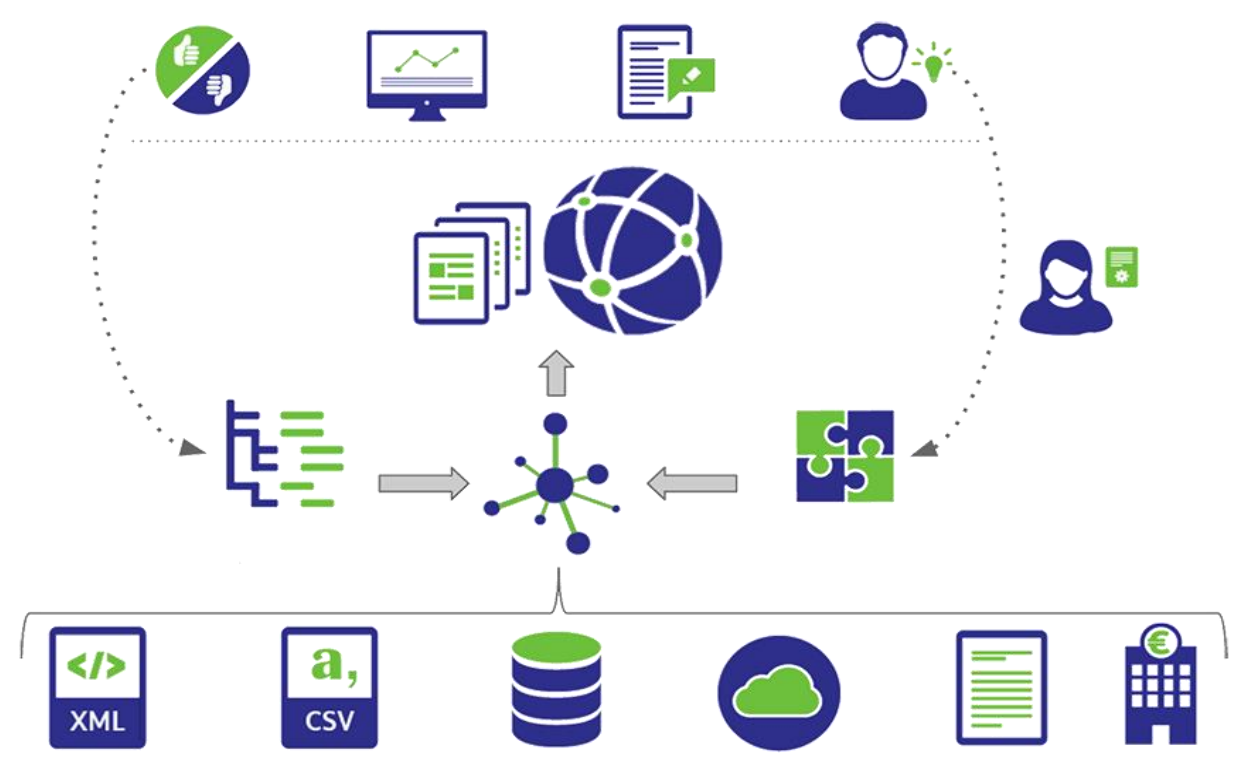

Figure. 1 Data Classification example

The same task can be implemented for an object or thing or a product in the market for marketing. The users may get time to time message son the data or issues related to that particular item can be discussed. The ideas or the opinions that were identified are of various forms like the good, positive, very good, very positive, negative, very negative and not recommended etc. Basically the sentences or the statements in the functions can be classified in to two types. They are objective and subjective. Based on the subject or based on the object these points are being classified of the form. The major marketing applications of these tasks are the Amazon, flipkart, mantra etc. Most of these applications will collect the data from various customers who had already purchased and using the products. Once the opinions are collected and the data of these reviews will be shared with the users of the same interest or interests on the same type of objects or the same type of products.

\subsection{Part of Speech Tagging}

The short form of the current application is POS tagging. The major tasks those were performed by using the current applications are the voice conversion, speech conversion, identification of the speeches, data and information conversion and extraction of data etc. 


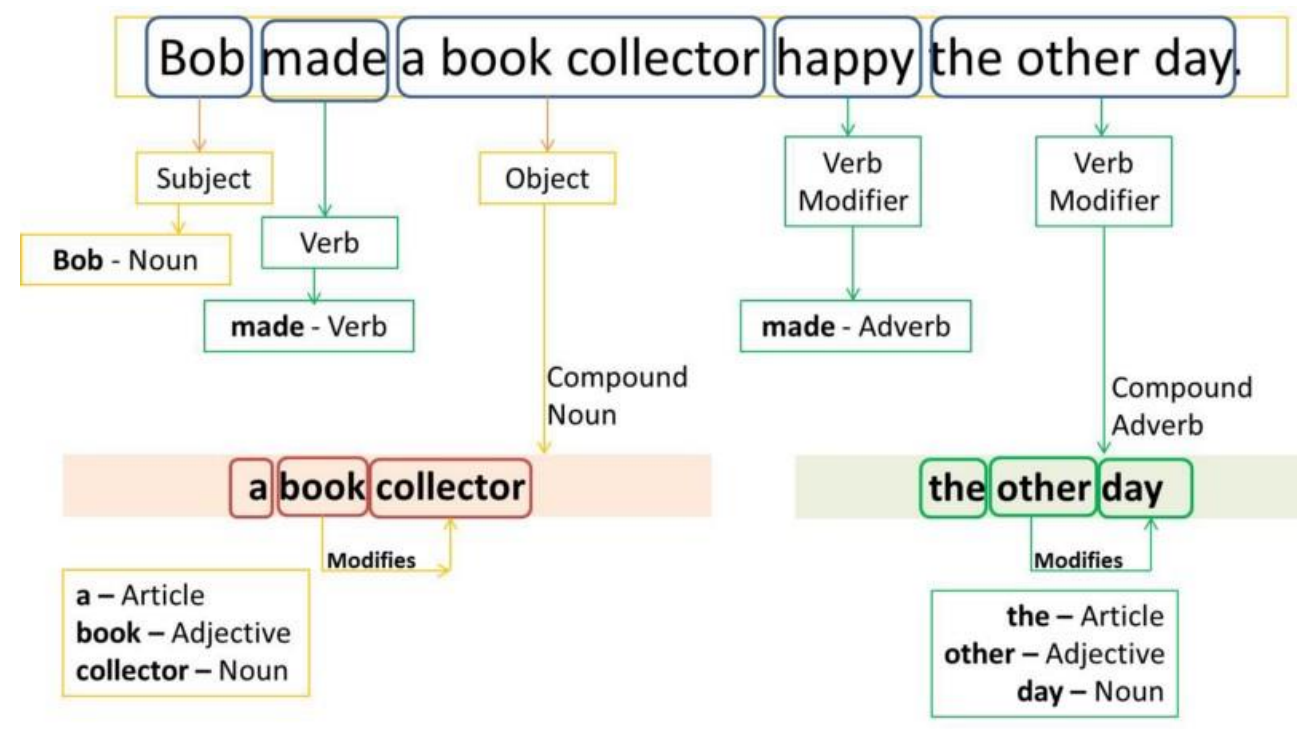

Figure. 2 Speech Tagging application example [2]

The current applications are mainly used in the officials meeting during the talks with other countries or the people who want to see the websites which were placed in their mother languages which were not English can be used such that to understand easily. If anybody want to see the websites of Chine or Russia, it is not possible to understand the local language, hence the people can use these sort of applications such that to convert or translate the local language to the standard understandable language English.

\subsection{Semantic based Question Answering}

The answering of the questions posed of various types of users either online or offline is most important. It plays a key role in identifying the data related to the question posed in different locations and the related data will be given by the form of answers to the users. Some of the questions include the meaning or the definitions of the questions, questions related to biography or the biographical questions, language based questions and performance based questions.

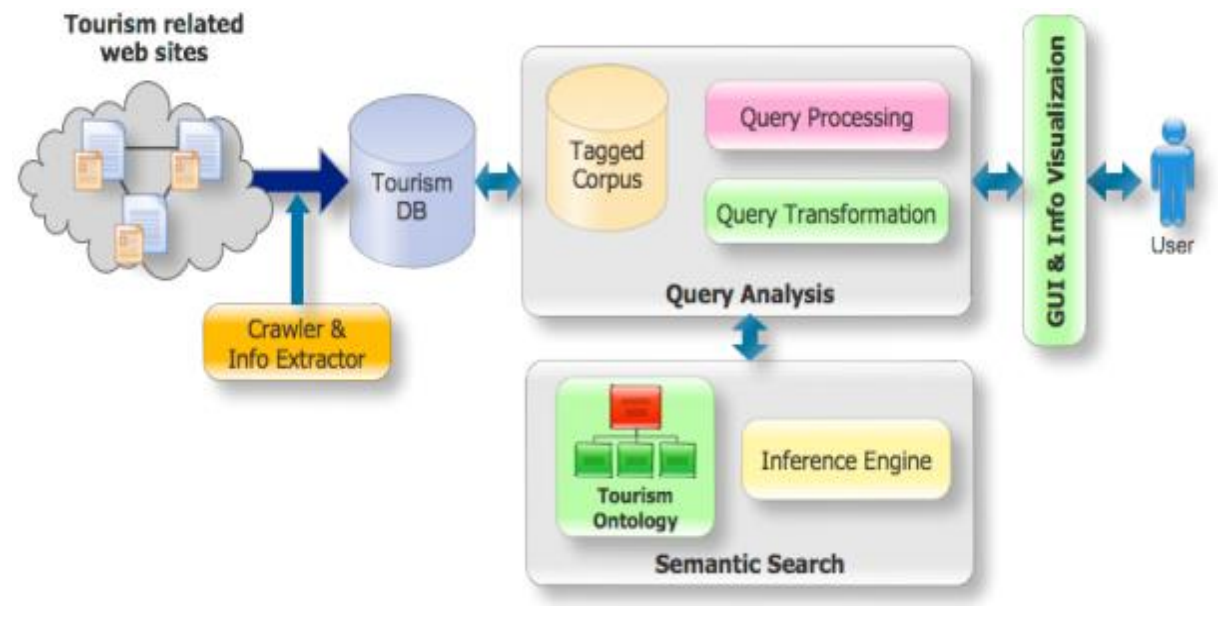

Figure .3 Semantic based question answering example [3] 
After the identification of the answers for the various set of questions that were raised during the time of the identification of the systems. Various set of machines like the rule based systems and the expert systems and their processing was also discussed and processed with the help of the data base or the knowledge base. In knowledge base, all the data required for the processing of these rule based systems or the inference based systems will be analyzed and given data for the further processing of the more and more number of machines and more amount of data.

\subsection{DATA DETECTION}

In the current data detection process, there are several ways or the methods to be performed such that to identify the data which was required or the data that was required to be submitted to some other machine as an input for further processing. The identification of similar data and answers for the similar type of questions was the next step that was processed in the form of data detection. This answering of questions those were raised during the processing of the applications because the questions may not be always asked in a similar way every time or the users may not pose the same question every time or in a similar way every time.

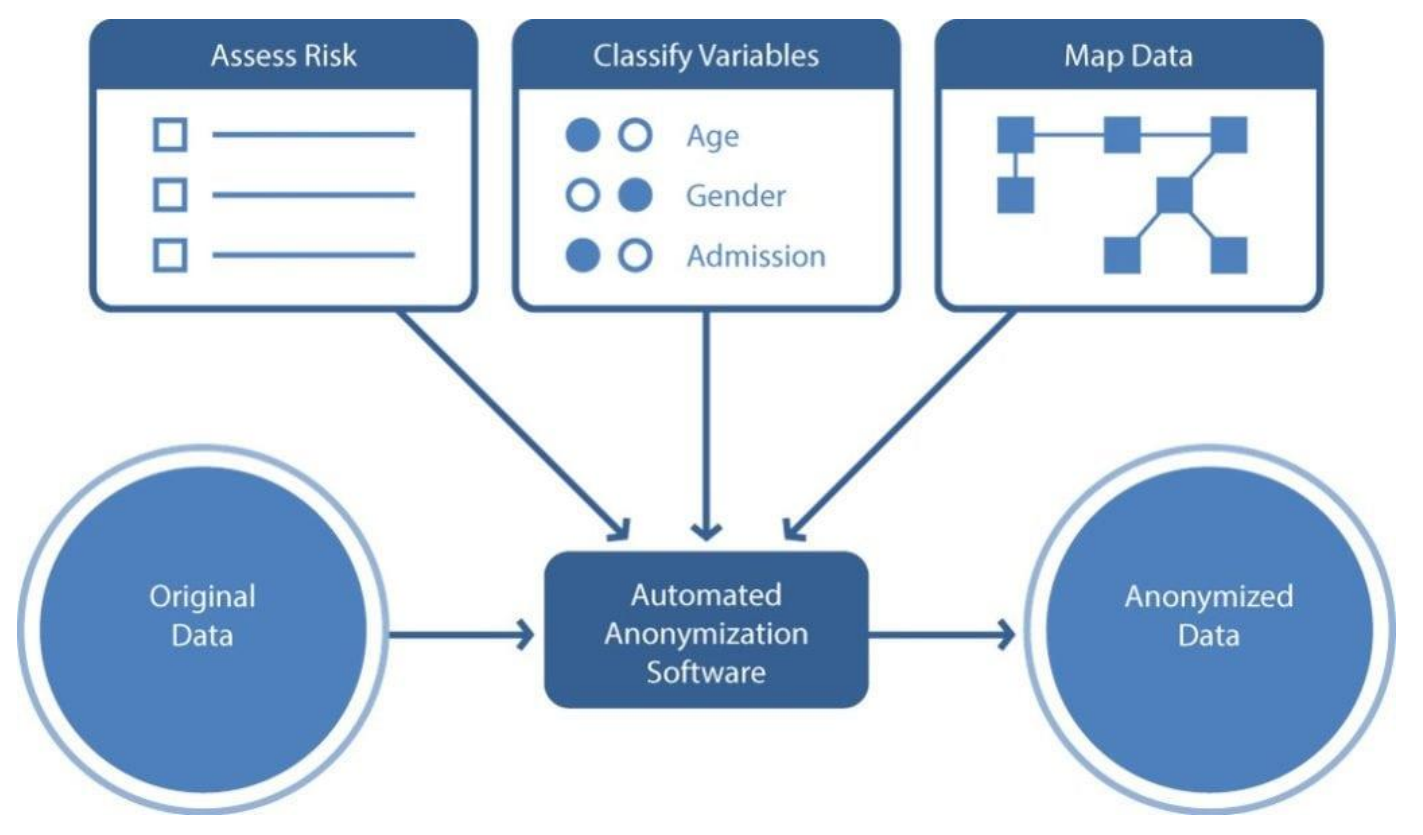

Figure. 4 Example of a data detection model [4]

Identifying the similar questions those were posed various types of users and their ages and their type of data browsing all those fields were collected and analyzed in detail. Once the data and its related topics are identified for almost all types of data, it is very easy for the users to process it and also for the further supplying of data to other set of users and their applications.

\subsection{Languages and Paragraphs Detection}

This application is very important and the reason for its importance is that the identification of various sentences and question having the same meaning is very important and difficult task too. It is very important due to the reason that always all questions in various languages may not be same or in reverse in some cases. The answers for the same type questions may not be same 
in almost all cases due to various reasons. The presence of language and its related things is also plays a vital role in various situations.

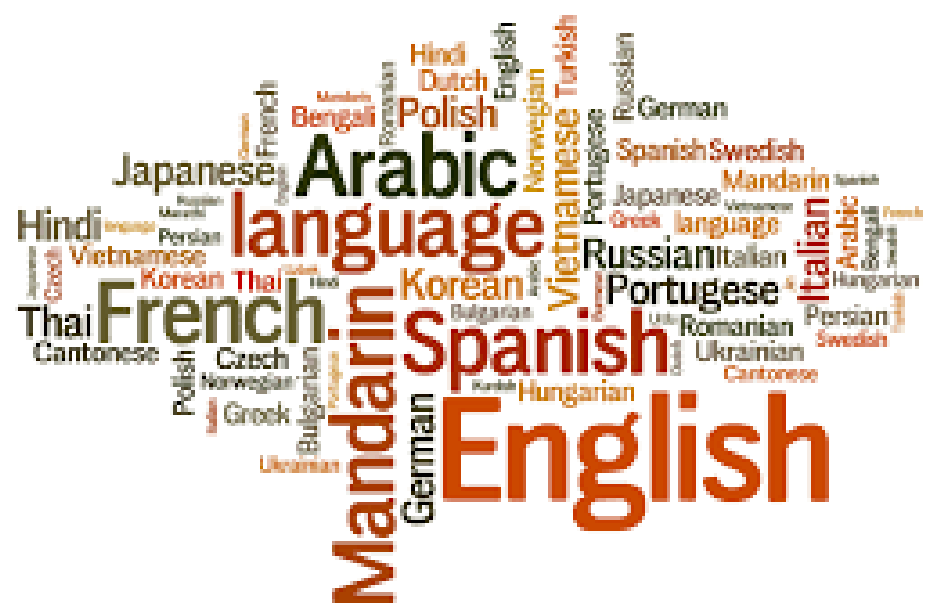

Figure. 5 Languages detection example [5]

Some of the techniques and methods to be used for identifying the languages and its similarity are the usage of Neural Network Models, Support Vector Machines, Autoencoder methods etc. One should have an good knowledge and good understanding about these methods and their implementation such that the data can be easily identified and processed for the better understanding $\mathrm{f}$ the questions and for finding the better answers for the raised questions on the way of finding the better results for the posed questions.

\subsection{TRANSLATION OF MACHINES DATA}

The translation of data or the information present in one form may be used for the same in other cases. It is different in various scenarios. It may change from one machine to the other machine because the entire machine may not work with similar types of software's or the operating system. As the operating systems changes, the data or the applications supported by those systems are also changes time to time. Hence, it can be very easy to process. 


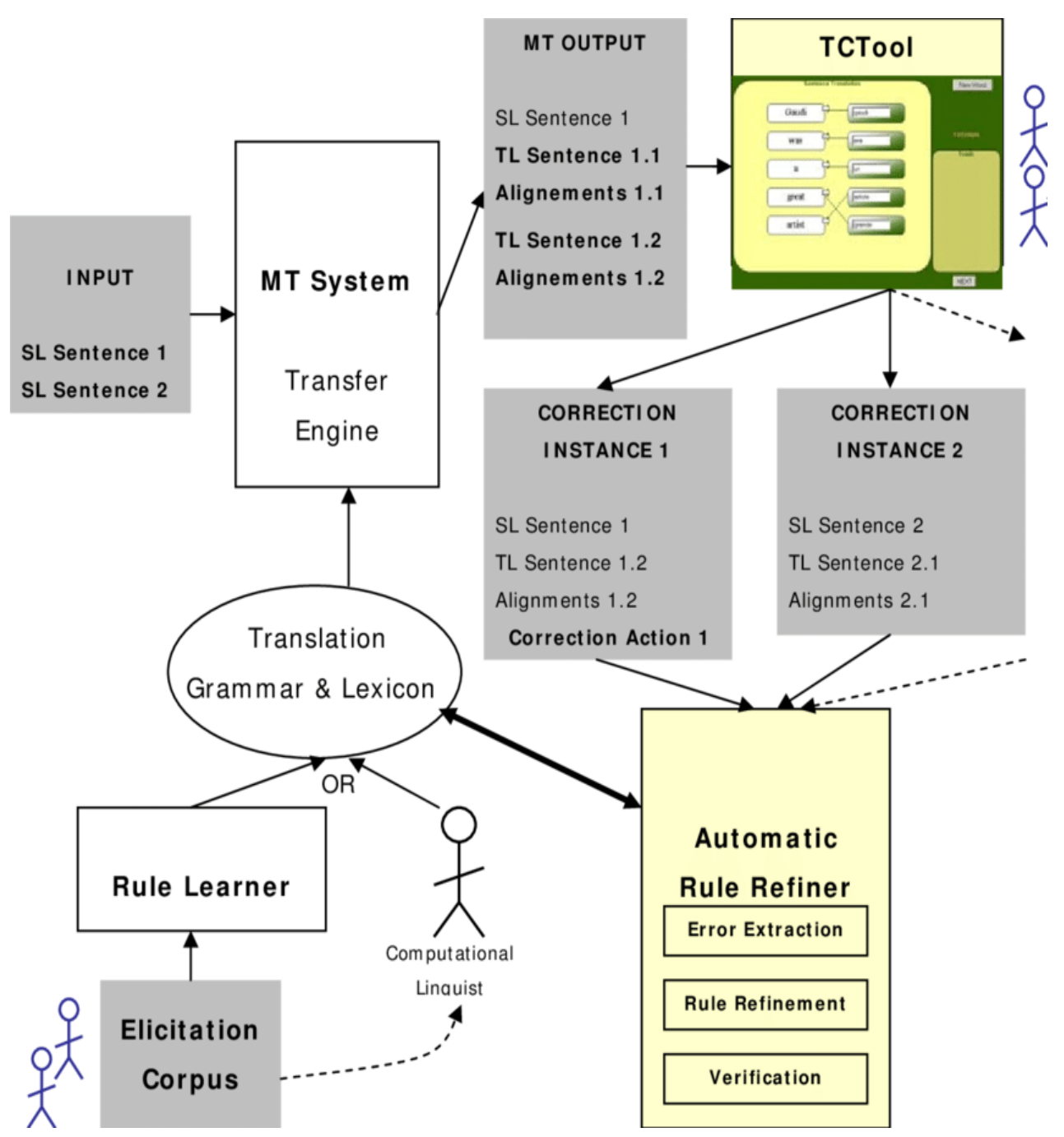

Figure.6 Machine data translation model example [6]

In earlier days, people use to stand beside with the others to listen and translate the same to the other person. But, in now a days as the technology grows and grows, the development of the further more technologies had made a rapid growth in the development of the current trends of applications. The machines are feuded with the language basics and the translator programming in such a way that all the topics and other issues related to the discussion were feuded and the machines will convert the required data whenever it is required to translate the data from one language to the other language.

\section{CONCLUSIONS}

In the current article an attempt has been made to discuss about the importance and working nature of both the areas like the neural networks, artificial neural networks and the natural language processing applications. All these topics are discussed in detail and the details were presented in the form of diagrams also. The current trends and the applications related to the natural language processing and the combination of this NLP with neural networks are also 
discussed in detail. The applications and their utility had also discussed in detail with the diagrams and another set of results also.

\section{References}

[1] https://bigid.com/ml-classification/ [last accessed on 10-06-(2019)]

[2] Sachin Malhotra and Divya Godayal, "An introduction to part-of-speech tagging and the Hidden Markov Model", Machine Learning, (2018).

[3] Alisa Kongthon, Sarawoot Kongyoung, Choochart Haruechaiyasak and Pornpimon Palingoon, "A Semantic Based Question Answering System for Thailand Tourism Information", Proceedings of the KRAQ11 Workshop, (2011), pp.38-42.

[4] https://privacy-analytics.com/de-id-university/how-to-become-a-data-de-identification-expert-learn-withhitrust-and-privacy-analytics/ [Last Accessed on 10-06-(2019)]

[5] Abhijeet Kumar, "Language Identification from Texts using Bi-gram model: Python/NLTK" in Machine Learning in Action (2017).

[6] Ariadna Font Llitjós, “Automatic Improvement of Machine Translation Systems”, submitted by to Carnegie Mellon University. (2007)

[7] https://medium.com/@datamonsters/artificial-neural-networks-in-natural-language-processing-bcf62aa9151a [Last Accessed on 10-6-(2019)]

[8] B Dinesh Reddy, A Ravi Kiran, Abhilash K, N.Thirupathi Rao and Debnath Bhattacharyya, "Solving VennDiagram Based Comprehension Questions in Set Theory using Natural Language Processing”, International Journal of Advanced Science and Technology, Vol.126, pp.11-20. (2019) 